\title{
Post Traumatic Epilepsy: A Review of Triggers and Potential Treatments after Brain Injury
}

\section{Emily McDonnell and Stephanie A Kolakowsky-Hayner}

Rehabilitation Research Center, Department of Physical Medicine and Rehabilitation, Santa Clara Valley Medical Center, San Jose, USA

\begin{abstract}
Posttraumatic epilepsy (PTE) is a significant and debilitating issue further complicating recovery after brain injury. This review is meant to be an overview of PTE following traumatic brain injury (TBI). Beginning with an introduction into the epidemiology of PTE following TBI, the paper goes on to explain the classifications of seizures and what defines posttraumatic epilepsy as well as the risks associated with the development of PTE after TBI. The biological basis of PTE is explained using both past and current research, assisting in understanding the past and current treatment options. This review concludes with a discussion of future research directions in order to improve upon the dearth of knowledge in this area and further build and establish well utilized treatment options.
\end{abstract}

Keywords: Posttraumatic epilepsy; Brain injury; Traumatic brain injury; Confusion; Consciousness

\section{Introduction}

Traumatic brain injury is the result of a blunt or penetrating force that creates symptoms including confusion, varying states of consciousness, coma, and focal sensory or motor deficits as well as seizures [1]. In 2010, the Center for Disease Control and Prevention reported that nearly 2.5 million traumatic brain injuries occurred either in isolation or in conjunction with another injury. The results and long-term effects of such injuries span a multitude of outcomes, with research focusing and acknowledging the increased likelihood of seizures following TBI. With such injuries comes the potential for the development of epilepsy, also referred to as posttraumatic epilepsy. Posttraumatic epilepsy is defined as two or more seizures, thought to be related to the injury itself, that follow a traumatic injury occurring later than one week following the injury $[2,3]$. Patients with TBI are at higher risk for the development of epilepsy relative to the general population. Posttraumatic epilepsy appears to account for up to $20 \%$ of symptomatic epilepsy within the general population [4]. As for the statistical likelihood of epilepsy in TBI patients, reports propose varying ranges with many resting anywhere from 3-20\% dependent on the severity of injury, which if severe, could increase the potential to approximately $40 \%[2,5,6]$.

There are variable pathophysiology's across TBI's that yield different outcomes in relation to seizures and the potential development of posttraumatic epilepsy (PTE). PTE in humans range from simple partial, complex partial to secondarily generalized seizures. The definitions of each, from the Epilepsy Foundation are as follows: simple partial, usually lasting around 2 minutes, is characterized as either motor, sensory, autonomic or psychic seizures in which the individual is usually alert. Complex partial seizures, lasting 1 to 2 minutes, include automatisms (such as lip smacking) in which the individual is unaware of their surroundings. Finally, secondarily generalized seizures begin in one area of the brain, and spread to both hemispheres lasting anywhere from 1-3 minutes. These seizures are convulsive and can take much longer for the patient to recover from. It is evident that these three types of seizures all exhibit different durations and causes, which are even further complicated by a patient's age, gender and even genetic background [7]. Factors such as age, previous seizures, smoking, genetics, timing of seizures and types of injury, all play a role in the potential for the development of PTE. All of these factors can create varying effects related to the outcome. For example, in pediatrics, the likelihood of late seizures is much lower than the likelihood of early seizures, which is often different from the adult population [2]. Age plays a role in the latency period of seizures following TBI. A latency period of around 3 years existed in those who were over 14 while there was an 18 year latency period in those who were 2 years or younger at the time of the trauma [8]. These vast differences further complicate risks and treatment. An additional risk factor that is often associated with an increased likelihood for the development of PTE, is genetic predisposition. Although much is still in debate as to what exactly may be the genetic markers, many articles propose that genetics could play a role in PTE $[9,10]$. Demonstrated through rats, it was demonstrated that regardless of the location and severity of the injury, other factors such as genetics or physiological states play a large role in the determination and the individual epidemiology of PTE [7]. This finding alone demonstrates why discovering treatments and solutions can prove to be so difficult.

\section{Classification and Risk}

The classifications of seizures following TBI are separated into three categories: immediate, early and late onset. Seizures may be related to the direct injury itself subsiding after hours or days, or long-term damage can occur, which often results in PTE. Each of these phases has separate definitions, causes and prognoses. Immediate onset seizures occur within 24 hours following the injury. These seizures are usually linked to the immediate repercussions of the injury as opposed to a change or alteration in neural circuitry. Most often these immediate seizures are seen within the pediatric population $[6,11,12]$. Immediate onset seizures are most often excluded from analysis in research, leaving their clinical significance unclear [2]. Early onset seizures typically occur less than a week after injury and are often seen as a precursor for late seizures $[6,13,14]$. Late onset seizures are found to occur more than a week after the injury and have been seen to occur for years following. These late seizures appear to increase the likelihood for PTE $[6,13]$.

*Corresponding author: Stephanie A Kolakowsky-Hayner, Santa Clara Valley Medical Center, 751 s. Bascom Avenue, San Jose, CA 95128, USA, Tel: 408 793-6446; Fax: 408-793-5434; E-mail: Stephanie.Hayner@hhs.sccgov.org

Received July 4, 2014; Accepted August 30, 2014; Published September 8, 2014

Citation: McDonnell E, Kolakowsky-Hayner SA (2014) Post Traumatic Epilepsy: A Review of Triggers and Potential Treatments after Brain Injury. Int J Neurorehabilitation 1: 115. doi:10.4172/2376-0281.1000115

Copyright: (C) 2014 McDonnell E, et al. This is an open-access article distributed under the terms of the Creative Commons Attribution License, which permits unrestricted use, distribution, and reproduction in any medium, provided the original author and source are credited. 
When comparing early versus late onset seizures, the different risk factors associated with each have been identified. Risk factors for early onset seizures comprise: acute intracerebral hematoma, acute subdural hematoma, younger age, increased injury severity, and chronic alcoholism [2]. Risk factors for late onset seizures include early posttraumatic seizures, brain contusion, subdural hematoma, prolonged posttraumatic amnesia ( $>3$ days), increased injury severity, and older than 65 years of age at the time of injury, along with many of the factors listed for early onset seizures $[2,4,6,15]$. Following a TBI, the likelihood of supplemental seizures is increased for up to 10 years following the injury [3], especially for injuries resulting in brain contusions and subdural hematomas. Rates of seizures following these injuries ranged from 5.0-12.0 as compared to only 2.2 for rate ratios from other injuries $[4,16]$. All of these injuries as well as outside factors play a role in differentiating the potential for PTE.

Most notable of all the previous risks, is the severity of the injury itself $[10,17]$. Common clinical markers of head injury severity include the Glascow Coma Score, length of posttraumatic amnesia, length of loss of consciousness, extent of brain damage and brain volume loss shown on CT scan [1,2]. As shown by Lowenstein, the risks for developing epilepsy based on severity are demonstrated as a 29 fold increased risk following TBI [6]. It is explained that there is a 4 times greater risk of developing epilepsy following moderate TBI as compared to a 1.5 times risk following a mild TBI [16]. Similar to this, penetrating versus closed head injuries damage the brain differently, leaving an altered likelihood for PTE. While closed head injuries often lead to axonal tearing, diffuse edema and ischemia, causing the release of excitatory amino acids and other toxic chemicals that can cause additional damage, penetrating injuries produce a cicatrix in the cortex, increasing the likelihood of PTE by about $50 \%$ versus $30 \%$ for the closed head injuries [4]. Additionally, severity appears to be related not only to a greater risk of seizures, but also a greater risk for a longer amount of time [14]. Therefore patients with highly severe brain injuries, were more likely to develop PTE and this likelihood was increased for a longer period of time. Often, PTE itself does not surface for months or even years following the injury [13] creating further questions as to the biological cause of the seizures themselves.

\section{Biological Basis}

As for the biological basis of PTE, numerous studies have noted various causes. There are still debates as to the exact causes leading to PTE, making treatment a challenge. Signs such as mossy fiber sprouting [18-20], increased electroencephalogram activity [11], damage to the hippocampus or cortex $[12,18]$, neuronal cell loss [18], blood brain barrier disruption $[13,21]$, inflammation $[7,20]$ and more appear to be potential precursors to the development of PTE.

Previously, findings indicated that there were two patterns of cell loss associated with TBI and the onset of seizures; these being classic hippocampal sclerosis and restricted hilar neuron loss [18]. Late seizures, which have been noted as a risk factor for epilepsy, are seen to be due to the damage of the cortex by free radicals that have been generated following the deposit of iron and the increased toxic level of glutamate [4]. Previous studies have also proposed that the reorganization of the circuitry in the brain can lead to epileptogenesis which is defined as the process by which a non-epileptic brain transforms into one that produces unprovoked seizures through the development of brain tissue capable of seizures [19]. The new connections made create hyperexcitability between the newly formed synapses particularly in the denate gyrus [22]. An increased electrical current appears to create the potential for epileptic activity. Mossy fiber sprouting itself is not the direct cause of epilepsy, but it is these increased connections on the dentate granule cells that further increases the excitability of the hippocampus $[12,13,18,19,23]$.

A more recent report summarizes some of the potential biological changes into three distinct temporal phases that occur after the injury. The first is in the early stage, ranging from a few seconds to minutes, in which there are changes observed in the ion channel related proteins and receptors. These changes create the beginning alterations to the proteins and genes which result in posttranslational modifications of both receptors and ion-channel proteins. Within hours to days there can be neuronal death, inflammation, and altered transcription factors associated with genes. These changes can lead to alterations in growth factors that begin the biological changes, as a precursor to the next phase. The later phase, which occurs weeks to months after injury often consists of morphological changes including mossy fiber sprouting, neurogenesis and gliosis [19,20]. All of these factors are seen to be biological markers for the development of PTE.

Additionally, the blood- brain barrier (BBB) is a more current biological topic of concentration to describe PTE after TBI. One study focused on the level and intensity of BBB disruption and its eventual effects on the potential of PTE. It was concluded that patients who developed PTE, had increased and higher volume of BBB disruption compared to non-PTE patients. Altogether they found that TBI patients showed a lasting focal increase in BBB activity in nearly $70 \%$ of the patients, a correlation between the EEG abnormality and the lesion, increased EEG slowing, correlation with the size of the BBB disruption to the extent of dysfunction, and patients with PTE were more likely to show abnormal BBB disruption overall compared to non-PTE patients [13]. BBB disruption and inflammation appear to be bi-products of TBI that often lead to further damage and disruption in the brain, hypothetically having an effect on PTE [21]. These biological factors provide a deeper understanding of the multiple factors playing a role in brain injury and the further understanding of this can truly assist in the treatment process.

\section{Treatment}

In reviewing the literature, current lines of treatment are structured in a step-wise process beginning with Anti-Epileptic Drug (AED) treatment, then moving to resection surgery if AED treatment is not ultimately effective and on to vagus nerve and deep brain stimulation for medically refractory epilepsy. Alternative approaches and therapies, such as hypothermia therapy, are also proposed as a potential new treatment as we will discuss further. As for the research on AED's, past treatment has focused on phenytoin prophylaxis.. Reports and success rates on this drug do not appear beneficial in preventing or treating later seizures. The success rates of phenytoin are limited to the first year, early seizures. These findings do not translate to the late unprovoked seizures or the further treatment of PTE $[5,9,11,13,15,24,25]$. In another article, comparing more AED drugs, using data on five separate AED drugs, including: phenytoin, valproate, phenobarbital, magnesium, and carbamazepine, researchers concluded that not one of the drugs has appeared to show lasting preventative effects on late seizures $[9,25]$. This review indicates that phenytoin and carbamazepine both have been seen to assist early seizures, but the same results, as previous studies have indicated, do not transfer to late seizures [25]. This finding demonstrates the need for additional treatment options for individuals who develop PTE. One study did find potential success in utilizing AED's. This study proposed a time window for the administration of AED's. This time window was within the first hour following a trauma, as this window can potentially protect against the underlying processes 
that lead to epileptogenesis [24]. Although medication is a more common form of treatment, the effectiveness should be called into question as many patients, despite taking medications report frequent seizures and difficulty with side effects when taking these drugs [26]. The proposal of a time window for the administration of AED's is an interesting aspect to be explored, but the window creates potential difficulties in attempting to determine the patients who would benefit from AED's while trying to save their lives. Additionally, articles have noted that many new anti-epileptic drugs have come onto the market, but have remained relatively unstudied; presenting a need for increased research as to the potential for improved benefits for these new drugs including gabapentin, rufinamide, lamotrigine and more [9]. The treatments that follow after the preliminary use of AED's are:

- Resection surgery of the anterior temporal lobe on the nondominant side [9]

- Deep Brain Stimulation [9]

- Vagus Nerve Stimulation [27]

- Hypothermia Therapy [23]

These treatment methods exhibit different yet promising benefits for the control and potential treatment of PTE. With the first avenue of treatment primarily headed by AED drugs, the second step is most commonly resection surgery. Resection surgery is utilized in patients in which genesis of the seizure is local and focused [9]. For instance, success has been demonstrated following resection surgery in patients with medial temporal lobe epilepsy, a more common location of epilepsy for patients with PTE [28]. Yet for PTE in which seizures can have multiple cortical epileptic foci and therefore resection is not an option, other strategies such as deep brain stimulation and vagus nerve stimulation may be utilized $[9,29]$. Both vagus nerve and deep brain stimulation are found to be effective for patients with medically refractory epilepsy [9]. In vagus nerve stimulation, an electrode is implanted onto the left vagus nerve, over time decreasing the amount, and potentially the severity of seizures, appearing to be a great potential treatment option for individuals experiencing PTE [27]. In a longitudinal study measuring the effectiveness of vagus nerve stimulation, Kuba et al. determined, after a 5 year follow up, that there was a $55.9 \%$ reduction in seizure frequency per month for patients [29]. Vagus nerve stimulation has indeed proved successful over the years for cases in which resection is not an option, but often stimulation is accompanied by AED treatment. Despite success with stimulation, most of the patients remained on AED treatment and it is with the addition of vagus nerve stimulation that seizure reduction was observed [29], As for deep brain stimulation, results vary, but in some cases suppression of seizures has been noted [30]. Deep brain stimulation has proven successful for Parkinson patients leading to the question of the same applicability to patients with PTE. Often deep brain stimulation is considered after resective surgery and/or vagus nerve stimulation, have failed [31]. Depending on the individual, there may be a personalization of treatment, but overall, there is no universal treatment.

Further delving into the methods mentioned above, some studies indicate promise within hypothermia therapy. Hypothermia therapy is defined as a modest reduction in brain temperature for only 4 hours after injury. This therapy appears to be a potential new treatment for PTE as some success has been shown in both the reduction of mossy fiber sprouting in the dentate gyrus as well as an overall reduction in the susceptibility to seizures. While this treatment was promising in some aspects, there were others that lacked statistically beneficial contributions. Hypothermia therapy did not reduce the loss of cells within the dentate gyrus and also did not decrease the severity of the seizures [23]. This led to the later proposal that hypothermia therapy be accompanied by a pharmacological approach that target the aspects such as hilar cell loss, that hypothermia therapy does not specifically target $[23,27]$. Hypothermia therapy exhibits varying successes, but with further research and trials, there may be greater breakthroughs allowing utilization for this type of therapy within the treatment process, especially if accompanied by pharmacological components.

Although these techniques all show promise, there is further research required for improvement of outcomes and possible prevention of PTE. Additionally, although promise has been shown on different treatment models, most experimentation was done on animals so new testing is required to see how the treatment would translate to the human population. Additionally, in considering these new treatment options, it has been noted in various studies, that these treatments are accompanied and bettered by the use of AED treatment, which is something that must be further considered and researched in the future $[23,27,31]$.

\section{Future Directions}

Thus far, many clinical trials targeted toward the prevention of epilepsy have ultimately failed [25] though there is promise amidst many of them. Future research is required for the discovery of factors that may be predictive in the development of PTE. This can be improved first by additional imagining and EEG observation in order to monitor the biological changes within the brain. Many biological factors mentioned above can be seen through imagining such as fMRI, thus, more observations could be a gateway toward learning new techniques and preventative measures. Studies have indicated that monitoring within the years following a TBI are crucial $[17,19]$; especially in the treatment and even discovery for the prevention of PTE. Additionally, many of these studies showing promise have solely been conducted on animals, thus more transitional studies and clinical trials are required in order to determine the success rates for humans [9]. Overall further research and observation is required in order to gain additional knowledge and treatment options for patients after TBI.

\section{References}

1. Bruns J, Hauser, WA (2003) The epidemiology of traumatic brain injury: A review. Epilepsia 44: 2. doi:10.1046/j.1528-1157.44.s10.3.x

2. Frey LC (2003) Epidemiology of posttraumatic epilepsy: a critical review. Epilepsia 44 Suppl 10: 11-17.

3. D'Ambrosio R, Perucca E (2004) Epilepsy after head injury. National Institute of Health 17: 731-735. [PubMed]

4. Agrawal A, Timothy J, Pandit L, Manju M (2006) Post-traumatic epilepsy: an overview. Clin Neurol Neurosurg 108: 433-439.

5. Yarnell S (1992) Physical medicine and rehabilitation. Epitomes. 663.

6. Lowenstein DH (2009) Epilepsy after head injury: an overview. Epilepsia 50 Suppl 2: 4-9.

7. Curia G, Levitt M, Fender JS, Miller JW, Ojemann J, et al. (2011) Impact of injury location and severity on posttraumatic epilepsy in the rat: role of frontal neocortex. Cereb Cortex 21: 1574-1592.

8. Manaka S, Takahashi H, Sano K (1981) The difference between children and adults in the onset of post-traumatic epilepsy. Folia Psychiatr Neurol Jpn 35: 301-304.

9. Chen JW, Ruff RL, Eavey R, Wasterlain CG (2009) Posttraumatic epilepsy and treatment. J Rehabil Res Dev 46: 685-696.

10. Christensen J, Pedersen MG, Pedersen CB, Sidenius P, Olsen J, et al. (2009) Long-term risk of epilepsy after traumatic brain injury in children and young adults: a population-based cohort study. Lancet 373: 1105-1110. 
Citation: McDonnell E, Kolakowsky-Hayner SA (2014) Post Traumatic Epilepsy: A Review of Triggers and Potential Treatments after Brain Injury. Int J Neurorehabilitation 1: 115. doi:10.4172/2376-0281.1000115

11. Rinaldi A, Conti L (2003) Posttraumatic epilepsy. Neurol Sci 24: 229-230.

12. Algattas H, Huang JH2 (2013) Traumatic Brain Injury pathophysiology and treatments: early, intermediate, and late phases post-injury. Int J Mol Sci 15: 309-341.

13. Tomkins O, Feintuch A, Benifla M, Cohen A, Friedman A, et al. (2011) Bloodbrain barrier breakdown following traumatic brain injury: A possible role in posttraumatic epilepsy. J Neurol Neurosurg Psychiatry 774-777.

14. Annegers JF, Coan SP (2000) The risks of epilepsy after traumatic brain injury. Seizure 9: 453-457.

15. Beghi E (2003) Overview of studies to prevent posttraumatic epilepsy. Epilepsia 44 Suppl 10: 21-26.

16. Annegers JF, Hauser WA, Coan SP, Rocca WA (1998) A population-based study of seizures after traumatic brain injuries. N Engl J Med 338: 20-24.

17. Bushnik T, Englander J, Wright J, Kolakowsky-Hayner SA (2012) Traumatic brain injury with and without late posttraumatic seizures: what are the impacts in the post-acute phase: a NIDRR Traumatic Brain Injury Model Systems study. J Head Trauma Rehabil 27: E36-44.

18. Swartz BE, Houser CR, Tomiyasu U, Walsh GO, DeSalles A, et al. (2006) Hippocampal cell loss in posttraumatic human epilepsy. Epilepsia 47: 13731382.

19. Jensen FE (2009) Introduction. Posttraumatic epilepsy: treatable epileptogenesis. Epilepsia 50 Suppl 2: 1-3.

20. Hunt RF, Haselhorst LA, Schoch KM, Bach EC, Rios-Pilier J, et al. (2012) Posttraumatic mossy fiber sprouting is related to the degree of cortical damage in three mouse strains. Epilepsy Res 99: 167-170.

21. Hunt RF, Boychuk JA, Smith BN (2013) Neural circuit mechanisms of posttraumatic epilepsy. Front Cell Neurosci 7: 89.
22. Cavazos JE, Cross DJ (2006) The role of synaptic reorganization in mesial temporal lobe epilepsy. Epilepsy Behav 8: 483-493.

23. Atkins CM, Truettner JS, Lotocki G, Sanchez-Molano J, Kang Y, et al. (2010) Post-traumatic seizure susceptibility is attenuated by hypothermia therapy. Eur J Neurosci 32: 1912-1920.

24. Benardo LS (2003) Prevention of epilepsy after head trauma: do we need new drugs or a new approach? Epilepsia 44 Suppl 10: 27-33.

25. Temkin NR (2009) Preventing and treating posttraumatic seizures: the human experience. Epilepsia 50 Suppl 2: 10-13.

26. Kolakowsky-Hayner SA, Wright J, Englander J, Duong T, Ladley-O'Brien S (2013) Impact of late post-traumatic seizures on physical health and functioning for individuals with brain injury within the community. Brain Inj $27: 578-586$.

27. Lee HO, Koh EJ, Oh YM, Park SS, Kwon KH, et al. (2008) Effect of vagus nerve stimulation in post-traumatic epilepsy and failed epilepsy surgery : preliminary report. J Korean Neurosurg Soc 44: 196-198.

28. Hartzfeld P, Elisevich K, Pace M, Smith B, Gutierrez JA (2008) Characteristic and surgical outcomes for medial temporal post-traumatic epilepsy. $\mathrm{Br} J$ Neurosurg 22: 224-230.

29. Kuba R, Brázdil M, Kalina M, Procházka T, Hovorka J, et al. (2009) Vagus nerve stimulation: longitudinal follow-up of patients treated for 5 years. Seizure 18: $269-274$

30. Tykocki T, Mandat T, Kornakiewicz A, Koziara H, Nauman P (2012) Deep brain stimulation for refractory epilepsy. Arch Med Sci 8: 805-816.

31. Ge Y, Hu W, Liu C, Zhang JG, Meng FG (2013) Brain stimulation for treatment of refractory epilepsy. Chin Med J (Engl) 126: 3364-3370. 